\title{
Verification of Paschen Law using a Mixed Geometry Disc- Conical Electrodes
}

\author{
Samah I. Radwan*, H. El-Khabeary ${ }^{\dagger}$, A.G. Helal ${ }^{*}$
}

\begin{abstract}
This work studies the Paschen law in electrical breakdown of a mixed geometry disc - conical electrodes from aluminum material at constant $10 \mathrm{~cm}$ distance using nitrogen gas. The discharge characteristices including discharge voltage, discharge current and breakdown voltage against the pressure-distance multiplied were measured at different pressures. The secondary electron emission coefficient was calculated using Townsend's secondary ionization coefficient equation. A comparison between two similar disc and conical structures with a mixed disc - conical structure was made for the breakdown voltage, discharge current and secondary electron emission coefficient. It is concluded that the values of secondary electron emission coefficient of conical cathode are higher than of disc cathode due to higher ions density on conical cathode than that disc one.
\end{abstract}

Keywords: Paschen Law, Secondary Electron Emission Coefficient, Mixed Disc and Conical Electrodes.

\section{Introduction}

The D.C gas discharge is used for many applications such as plasma display panels, lamps, surface modification, and biomedical applications, [1,2]. The electrical characteristics (I-V) are linear for most the gas discharge. The linear form indicates that the voltage across the plasma remains nearly constant while the current increases, [3]. Also the (I-V) characteristic shows that the potential between the two electrodes increases with the increase of the discharge current. The breakdown voltage increases with the decrease of working gas pressure, [4]. The secondary electrons emission from the cathode surface is very effective, where the (I-V) characteristics changes according it, [5].

In DC discharges, the breakdown is usually described by the standard Townsend's theory, [6]. It is represented by a Paschen curve in which the breakdown voltage, $\mathrm{V}_{\mathrm{b}}$, dependence on the multiplied of gas pressure, $\mathrm{P}$, and gap electrodes distance, $\mathrm{d}$, [7]. At high $\mathrm{Pd}$, due to a large number of collisions, $\mathrm{V}_{\mathrm{b}}$ is increased in order to enhance energy gain between collisions that the mean free path is getting shorter and the energy gained between two collisions becomes smaller. In the range of the Paschen minimum, the production of charges by ionization and secondary electron emission losses by attachment, diffusion and drift are well balanced.

\footnotetext{
Dr, Accelerators \& Ion Sources Department-Nuclear Research Center-Atomic Energy Authority-Egypt, P.N. 13759 Cairo, Egypt, torabsamah@yahoo.com

$\dagger \quad$ Prof., Accelerators \& Ion Sources Department-Nuclear Research Center-Atomic Energy Authority-Egypt, Head of Basic Nuclear Science Division, P.N. 13759 Cairo, Egypt, helkhabeary@yahoo.co.uk

* Prof., Accelerators \& Ion Sources Department-Nuclear Research Center-Atomic Energy Authority-Egypt, P.N. 13759 Cairo, Egypt, helaladel19@gmail.com
} 
Townsend first ionization coefficient, $\alpha$, is usually presented as similarity parameter, $\alpha / \mathrm{P}$, depending on the reduced electric field $E / P$. Where $\alpha / P=f(E / P)$ for different gases can be found, [8]. Each primary electron generated near a cathode produces [ $\exp (\alpha \mathrm{d})-1]$ positive ions moving back to the cathode. The ions lead to the extraction of $\gamma[\exp (\alpha \mathrm{d})-1]$ electrons from the cathode due to secondary electron emission characterized by the Townsend coefficient, $\gamma$.

\section{A. Townsend's coefficients}

Paschen law is used to describe the mechanism of gas breakdown, [9]. Townsend introduced Townsend's first ionization coefficient, $\alpha$, in order to explain the current increase as a function in the voltage drop, where the average current is equal to the number of electrons traveling per second and can be given by equation (1):

$$
I=I_{0} \cdot e^{\alpha d}
$$

where $I_{0}$ is the current at the cathode, $d$ is the electron traveled distance and $\alpha$ is the first Townsend's ionization coefficient.

The secondary electron emission coefficient, $\gamma$, plays an important role in determining the gas breakdown voltage as well as overall characteristics of the discharge. It provides the information on the efficiency of electron emission from the cathode due to ion bombardment. Emission by ions can be one of the major causes of electron emission, but it is never the only cause and not often dominant. To determine the effective coefficients, we will use the practical breakdown voltage, $[7,10,11]$. The theory for this calculation is based on $\gamma$ and $\alpha$ that the latter expresses the number of single ionizing collisions by an electron in unit distance. These two coefficients appear in the self-sustaining condition for a homogeneous electric field and their relation can be given in equation (2), $[12,13]$ :

$$
\gamma\left(e^{\alpha \mathrm{d}}-1\right)=1
$$

The first Townsend's ionization coefficient is the number of ionized ions by an electron through one centimeter distance. It depends on the gas pressure, or the gas density which is a function of collision mean free path, and the reduced electric field strength. The generalized form of $\alpha$ for planner diode can be expressed by equation (3), [13,14]:

$$
\alpha=\mathrm{A} P \exp [-\mathrm{B} \mathrm{P} / \mathrm{E}]
$$

Where $\mathrm{A}$ and $\mathrm{B}$ are constants dependence on the used gas and equal to 10.95, 273.78 for nitrogen gas respectively, $\mathrm{P}$ is the gas pressure and $\mathrm{E}$ is the electric field.

The secondary electron emission coefficient effect is significant especially during the breakdown stage where the number of electrons is so small that the gas phase reactions can hardly sustain the discharge. Hence the breakdown voltage, which is one of the most essential parameters in discharge devices, is critically affected by the secondary electron emission coefficient. By considering that before and at breakdown, the electric field is uniform along the discharge axis and from equations (2) and (3) we obtain:

$$
\gamma=1 /\left(e^{\alpha d}-1\right)=\left\{1 /\left[\exp \left(A P d \cdot e^{-\left(\frac{B P d}{V}\right)}\right)-1\right]\right\}
$$

where V is the voltage applied between two electrodes. From equation (4), we can calculate the secondary electron emission coefficient that defined as the number of electrons emitted from 
the surface for each incident ion or atom. The work function is the minimum energy required to remove an electron from a solid to point in the vacuum.

The random electrons produced by cosmic rays that can add to their number by ionizing gas molecules by collision. Each ionizing collision produces a new electron and a positive ion. The electron will migrate to the anode, the ion to the cathode and a small current will flow. An electron cannot do this unless it has acquired sufficient kinetic energy by being accelerated in an electric field that applied between the anode and the cathode. Electrons striking a metal surface can knock loose secondary electrons readily, but this is of little use for discharges, since the electrons impact the anode and secondary electrons would simply fall back into the anode. However, the positive ions can also create secondary electrons. Although this is not an efficient process, it produces electrons at the right place and can support a discharge.

\section{Experimental Setup}

Figure (1) shows Experimental setup of a mixed geometry disc-conical electrodes from aluminum material in the discharge tube. The anode - cathode is fixed at distance equal to 10 $\mathrm{cm}$ in a cylinder quartz tube of $50 \mathrm{~cm}$ length and $36.5 \mathrm{~cm}$ diameter. The two ends of this tube are closed by two cylindrical plastic insulators that prevent the gas leakage. One of them is mounted to the rotary pump to evacuate this tube up to $10^{-3}$ Torr and the other one is connected to the needle valve to control the nitrogen gas flow which feeding through a fine controlled gas admittance needle valve to control and adjust the gas flow rate. A digital vacuum meter type 1005 Edwards is connected to the pump to measure the pressure inside the tube. The discharge was operated using nitrogen gas of purity equal to $99.99 \%$ at typical pressure values in the range $0.1-0.6$ Torr. The disc electrode geometry was $7.5 \mathrm{~cm}$ diameter and $1.0 \mathrm{~cm}$ thickness. The conical electrode geometry was $5.0 \mathrm{~cm}$ diameter base and $5.0 \mathrm{~cm}$ height.

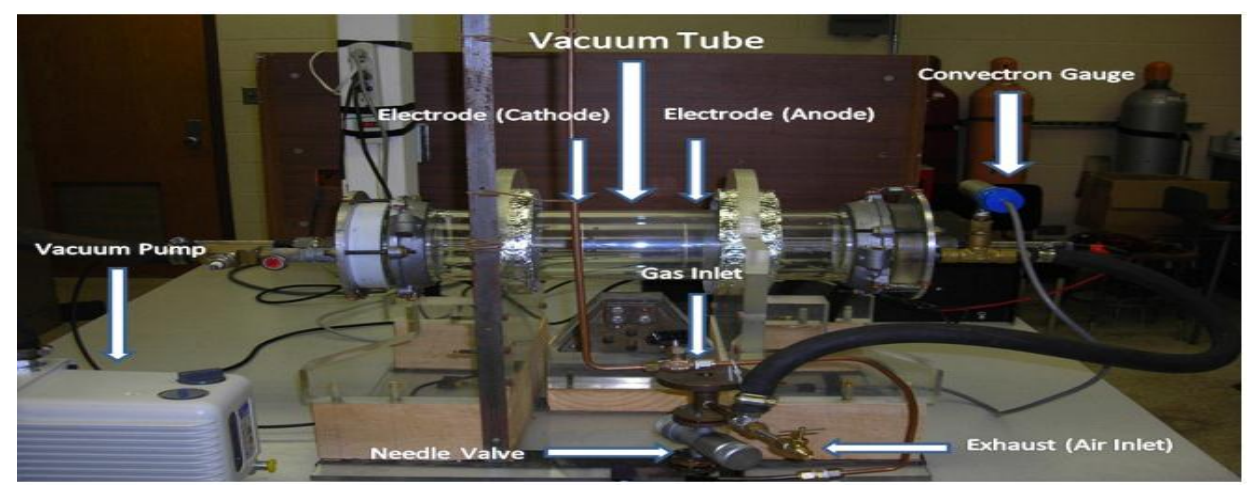

\section{Fig. 1. Experimental Setup of a Mixed Disc - Conical Anode and Cathode Electrodes.}

Electrical circuits for a mixed disc and conical anode - cathode from aluminum material as shown in figures $(2,3)$. A $5 \mathrm{kV}-10 \mathrm{~mA}$ digital Spellman type positive power supply of $\pm 3 \%$ accuracy is connected to the anode for initiating glow discharge and the cathode is connected to ground. A millimeter, $\mathrm{mA}$, with range $10 \mathrm{~mA}$ is used to measure the discharge current between the anode and cathode, while a voltmeter, $\mathrm{V}$, with range $0-5000 \mathrm{~V}$ used to measure the discharge voltage between them. 


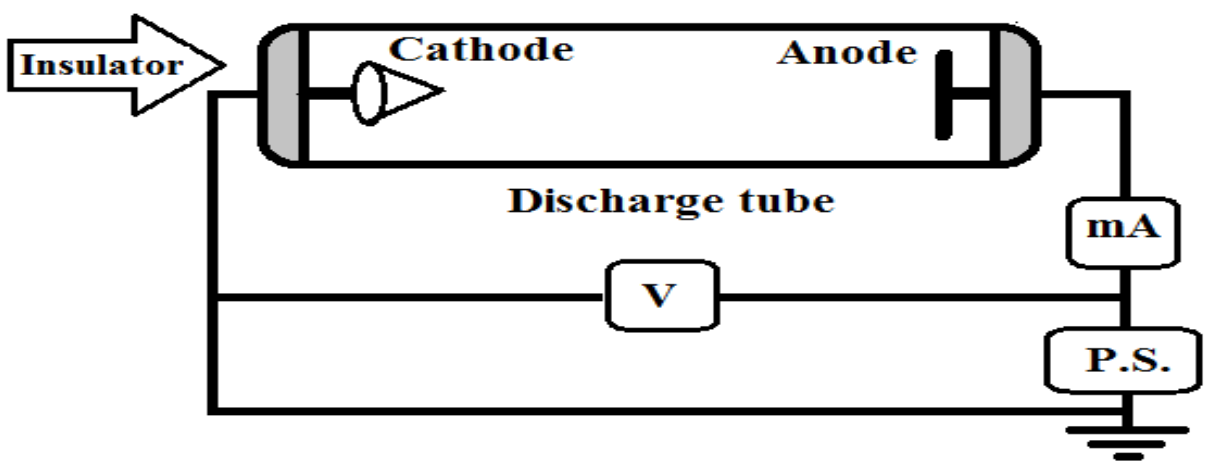

Fig. 2. Electrical Circuit for Disc Anode - Conical Cathode.

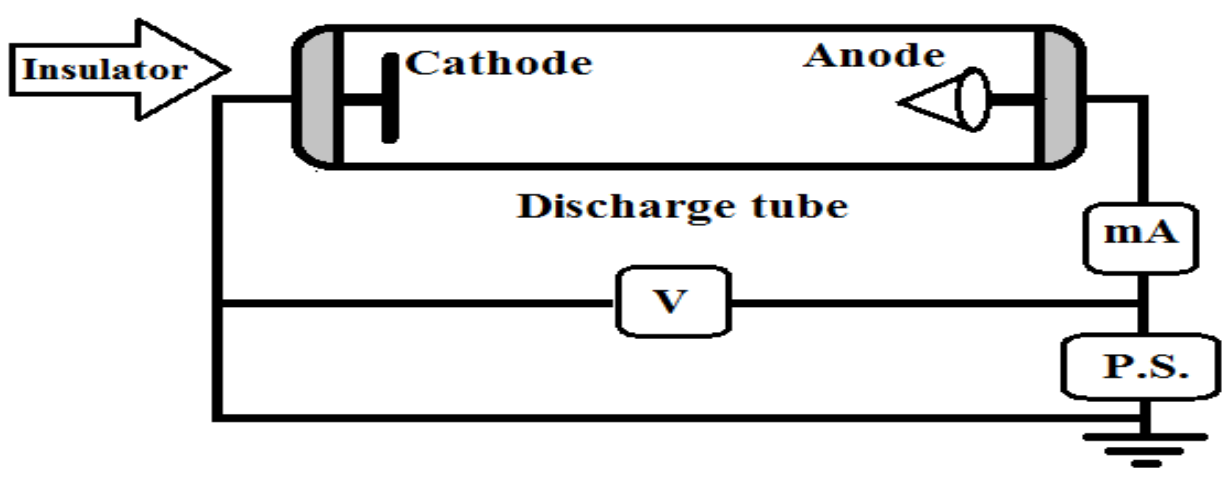

Fig. 3. Electrical Circuit for Conical Anode - Disc Cathode.

\section{Experimental Results}

The experimental results are measured for two cases that disc anode - conical cathode and conical anode - disc cathode from aluminum material. The disc has $7.5 \mathrm{~cm}$ diameter with 0.7 $\mathrm{cm}$ thickness while the cone has $5 \mathrm{~cm}$ height and $5 \mathrm{~cm}$ base diameter. The discharge voltage and current against the pressure - distance multiplied, $\mathrm{Pd}$, were measured at different pressures using nitrogen gas. The secondary electron emission coefficient was calculated using Townsend's secondary ionization coefficient equation.

\section{A. Discharge characteristics of mixed geometry disc-conical electrodes}

Figure (4) shows breakdown voltage, $V_{b}$, versus discharge current, $I_{d}$, for disc anode - conical cathode at different pressures using nitrogen gas at a distance of $10 \mathrm{~cm}$. It's obvious that the breakdown decreases by increasing the discharge current until reaching $444 \mathrm{~V}$ at $2.3 \mathrm{~mA}$, after that the discharge current increases linearly by increasing the discharge voltage and reaching $27.8 \mathrm{~mA}$ at $689 \mathrm{~V}$. It is seen that the discharge is of the abnormal glow one.

Figure (5) shows breakdown voltage, $V_{b}$, versus discharge current, $I_{d}$, for conical anode - disc cathode at different pressures using nitrogen gas at a distance of $10 \mathrm{~cm}$. It is clear that the breakdown voltage decreases by increasing the discharge current and reaching a minimum value equal to $447 \mathrm{~V}$ at $1.4 \mathrm{~mA}$, then the breakdown voltage increases by increasing the discharge current and reaching $968 \mathrm{~V}$ at $49.9 \mathrm{~mA}$. When the applied voltage is increased, the current throughout the gap increases slowly, as the electrons emitted from the cathode move through the gas with an average velocity determined by their mobility for the field strength existing for the particular value of voltage. Impact ionization by electrons is probably the most important process in the breakdown of gasses, but this process alone is not sufficient to produce breakdown. 


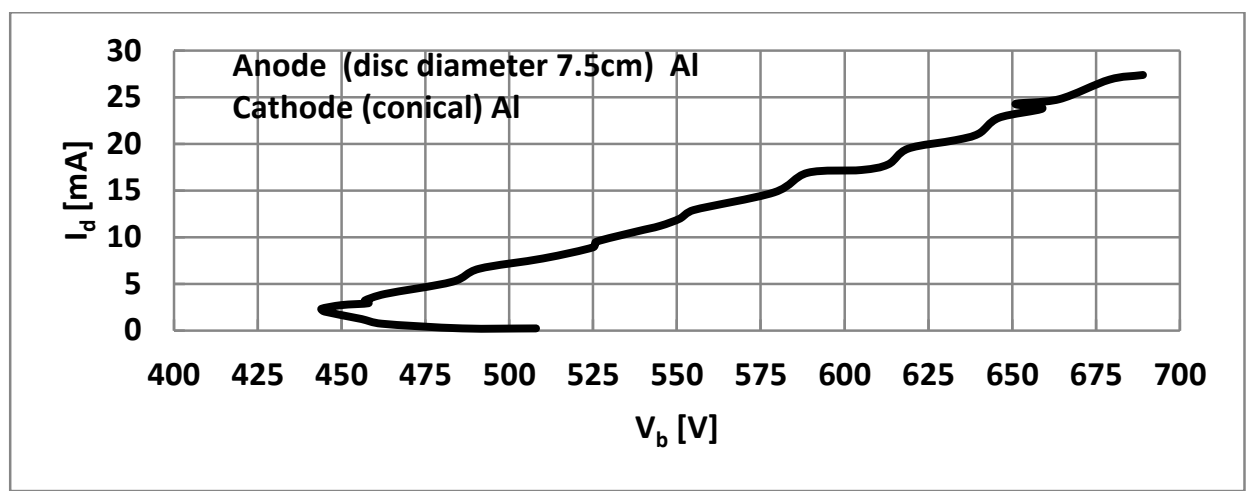

Fig. 4. Voltage - Current Characteristics for Disc Anode - Conical Cathode.

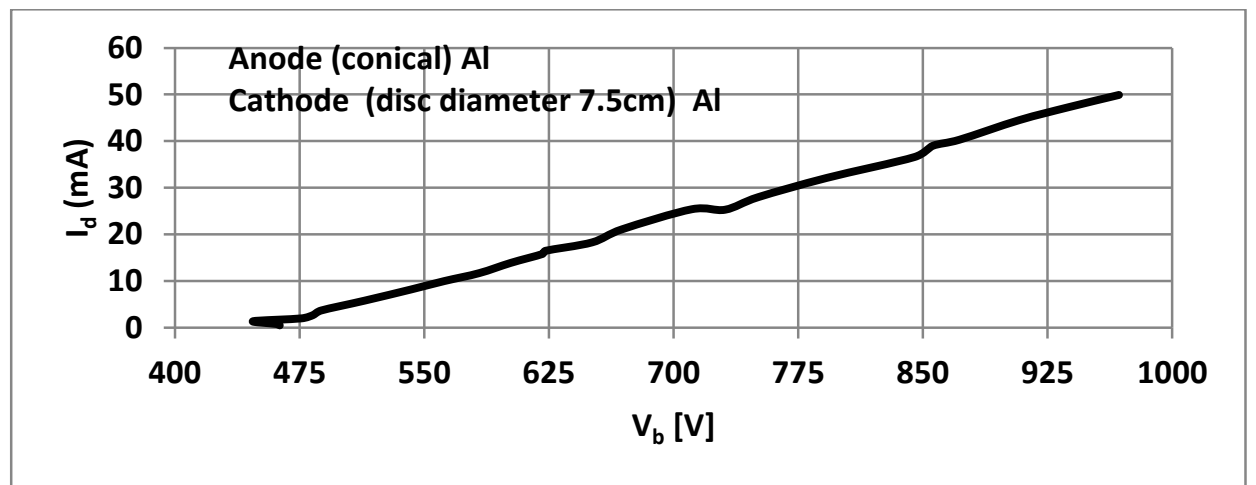

Fig. 5. Voltage - Current Characteristics for Conical Anode - Disc Cathode.

Figures $(6,7)$ show breakdown voltage, $V_{b}$, versus $P d$ (Paschen curve) for disc anode-conical cathode and conical anode-disc cathode at different pressures respectively. It is clear that the breakdown voltage decreases by increasing the nitrogen gas pressure and reaching a minimum value equal to $444 \mathrm{~V}$ and $476 \mathrm{~V}$ at $\mathrm{Pd}$ equal to 1.64 Torr.cm and 1.51 Torr.cm, then the breakdown voltage increases by increasing the nitrogen gas pressure and reaching $689 \mathrm{~V}$ and $968 \mathrm{~V}$ for conical cathode and disc cathode respectively.

When the applied voltage is increased, the current throughout the gap increases slowly, as the electrons emitted from the cathode move through the gas with an average velocity determined by their mobility for the field strength existing for the particular value of voltage. Impact ionization by electrons is probably the most important process in the breakdown of gasses, but this process alone is not sufficient to produce breakdown.

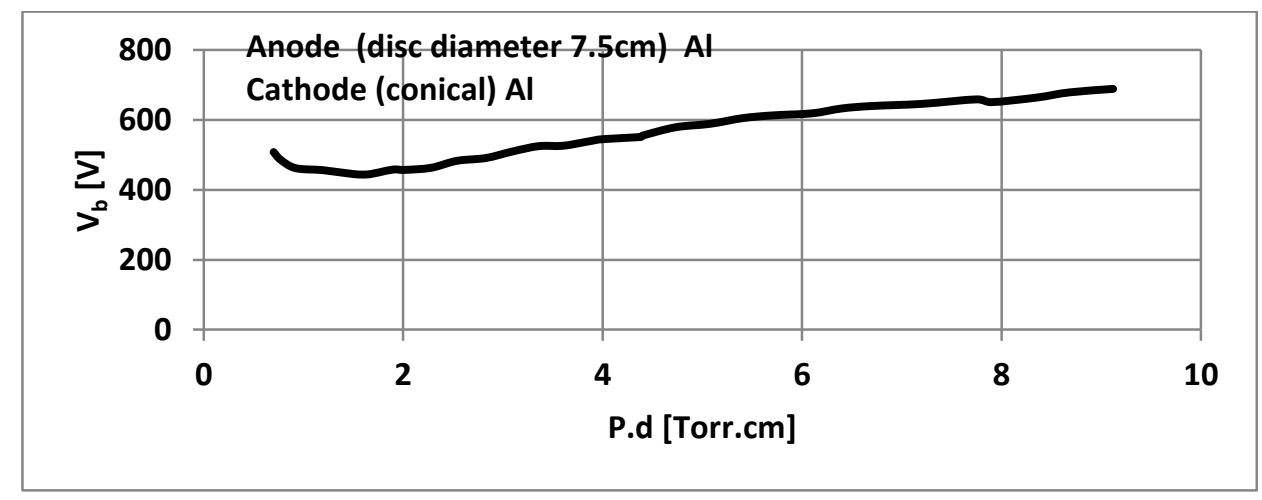

Fig. 6. Breakdown Voltage versus Pd for Disc Anode - Conical Cathode. 


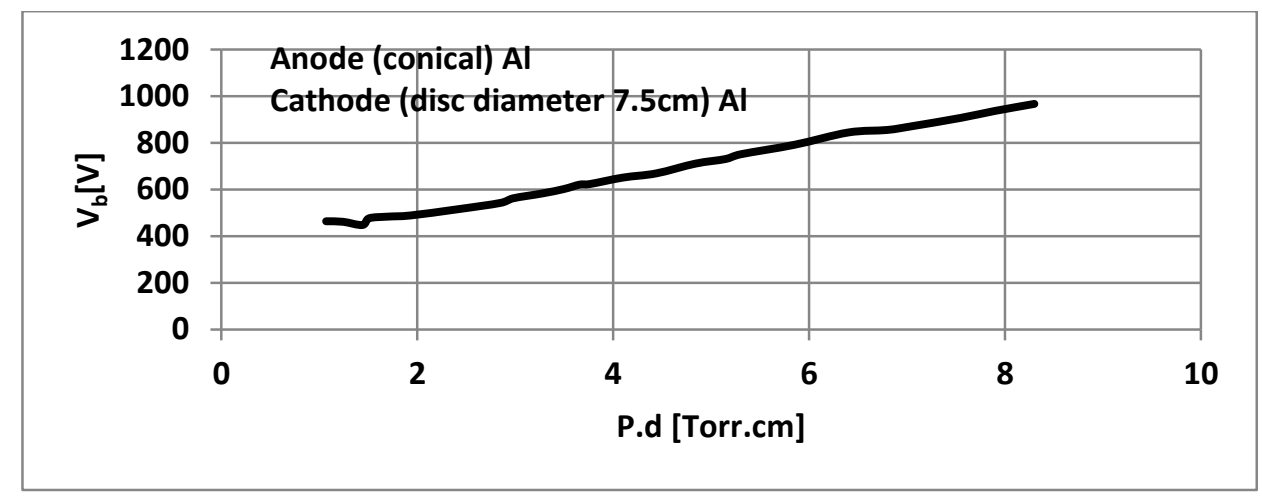

Fig. 7. Breakdown Voltage versus Pd for Conical Anode - Disc Cathode.

\section{B. Comparison between mixed geometry disc-conical electrodes}

A comparison of the breakdown voltage against the pressure - distance multiplied for mixed geometry disc-conical electrodes is made. Figure (8) shows comparison breakdown voltage, $\mathrm{V}_{\mathrm{b}}$, versus $\mathrm{Pd}$ (Paschen curves) for mixed geometry disc-conical electrodes at different pressures. It is clear that $\mathrm{V}_{\mathrm{b}}$ decreases by increasing nitrogen gas pressure and reaching a minimum breakdown values equal to $450 \mathrm{~V}$ and $430 \mathrm{~V}$ at constant Pd equal to 1.5 Torr.cm for discs and conical electrodes. Then it increases by increasing pressures and reaching $1000 \mathrm{~V}$ and $700 \mathrm{~V}$ for discs and conical electrodes at Pd equal to 8 Torr.cm and 9 Torr.cm respectively.

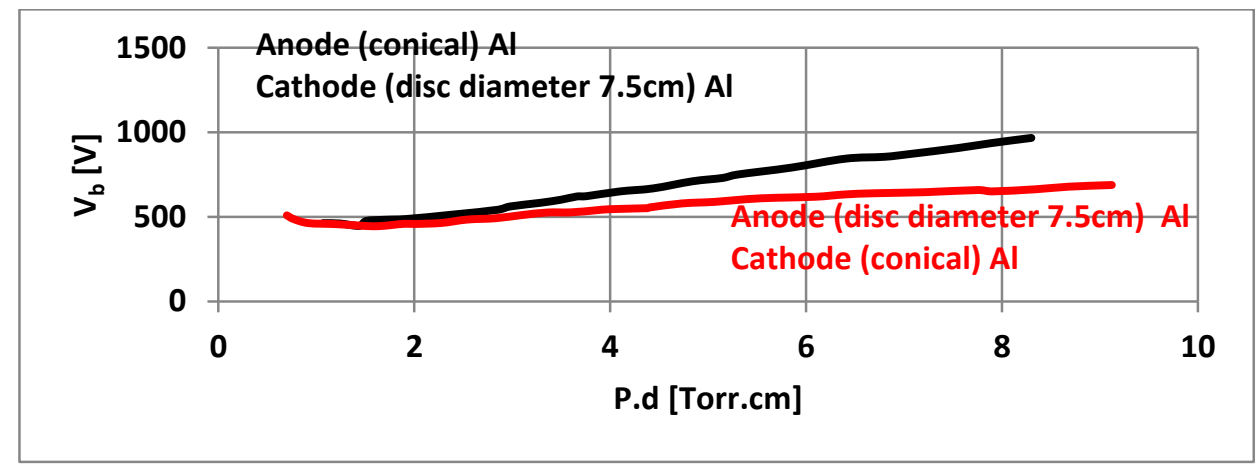

Fig. 8. Breakdown Voltage versus Pd for Mixed geometry Disc-Conical Electrodes.

Figures $(9,10)$ show discharge current, $I_{d}$, versus Pd (Paschen curve) for mixed geometry discconical electrodes at different pressures. It is clear that the discharge current increases by increasing the nitrogen gas pressure and reaching $27.4 \mathrm{~mA}, 49.9 \mathrm{~mA}$ at $\mathrm{Pd}$ equal to 9.12 Torr.cm, 8.3 Torr.cm for conical cathode and disc cathode respectively. 


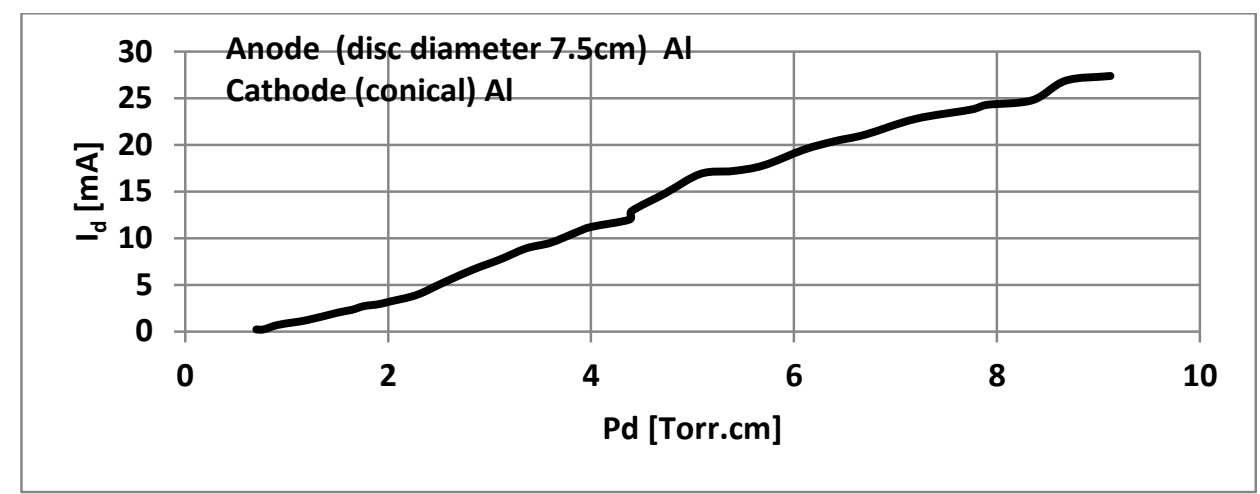

Fig. 9. Discharge Current versus Pd for Disc Anode - Conical Cathode.

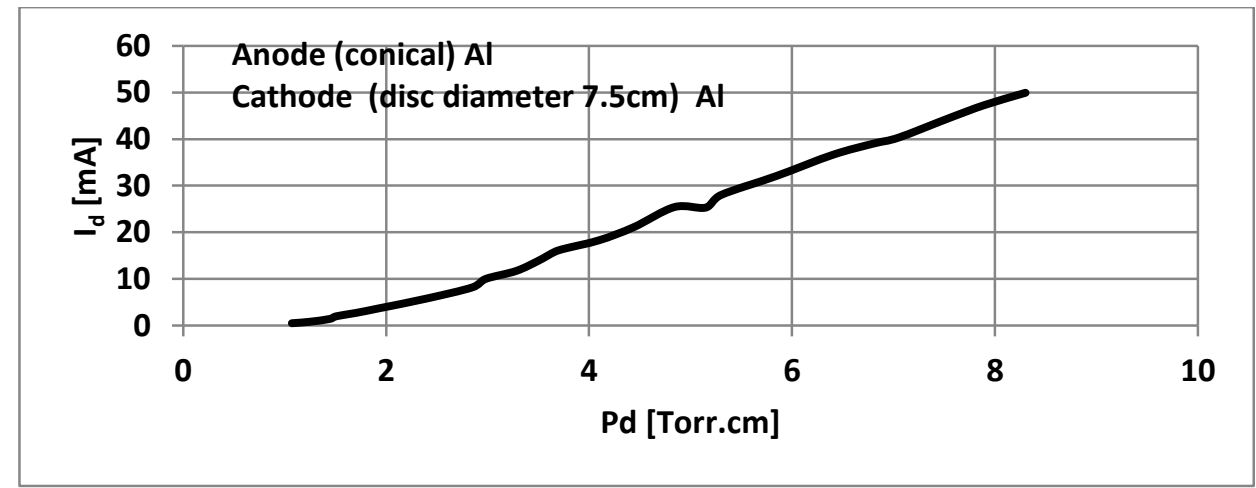

Fig. 10. Discharge Current versus Pd for Conical Anode - Disc Cathode

Figure (11) shows comparison of discharge current, $I_{d}$, versus Pd (Paschen curve) for mixed geometry disc-conical electrodes at different pressures. It is clear that the discharge current increases by increasing the nitrogen gas pressure and reaching $24.8 \mathrm{~mA}, 49.9 \mathrm{~mA}$ at constant Pd equal to 8.3 Torr.cm for conical cathode and disc cathode respectively.



Fig. 11. Comparison of Discharge Current versus Pd for Mixed Geometry DiscConical Electrodes. 


\section{Secondary electron emission coefficient of mixed conical aluminum anode - cathode electrodes}

Figures $(12,13)$ show secondary electron emission coefficient, $\gamma$, versus Pd for mixed geometry disc-conical electrodes at different pressures. It is obvious that $\gamma$ increases by increasing the nitrogen gas pressure until reaching 0.55 and 0.005 at pressure equal to 0.9 Torr and 0.8 Torr respectively.

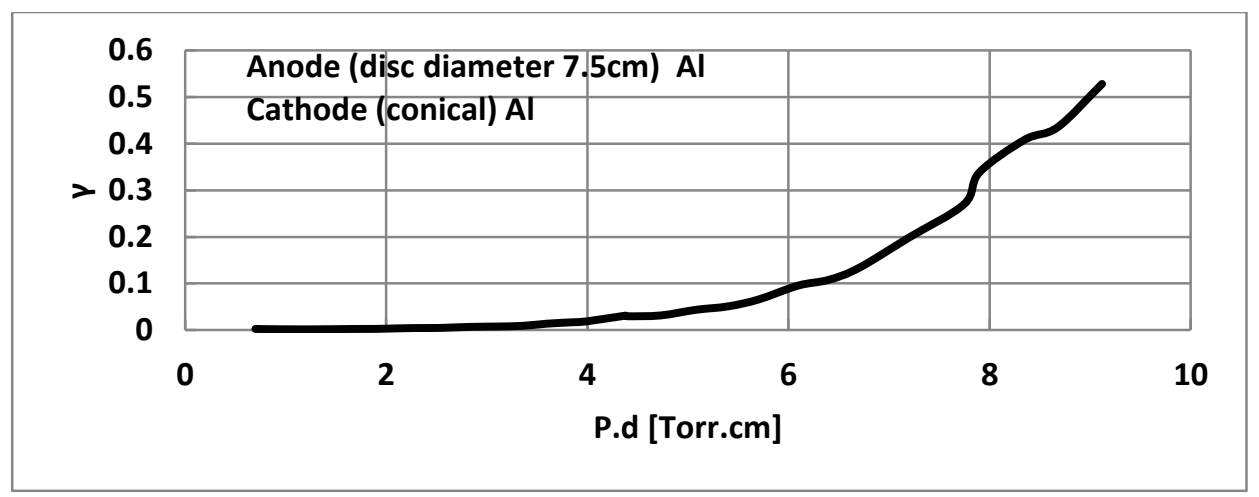

Fig. 12. Secondary Electron Emission Coefficient versus Pd for Mixed Geometry Disc-Conical Electrodes.

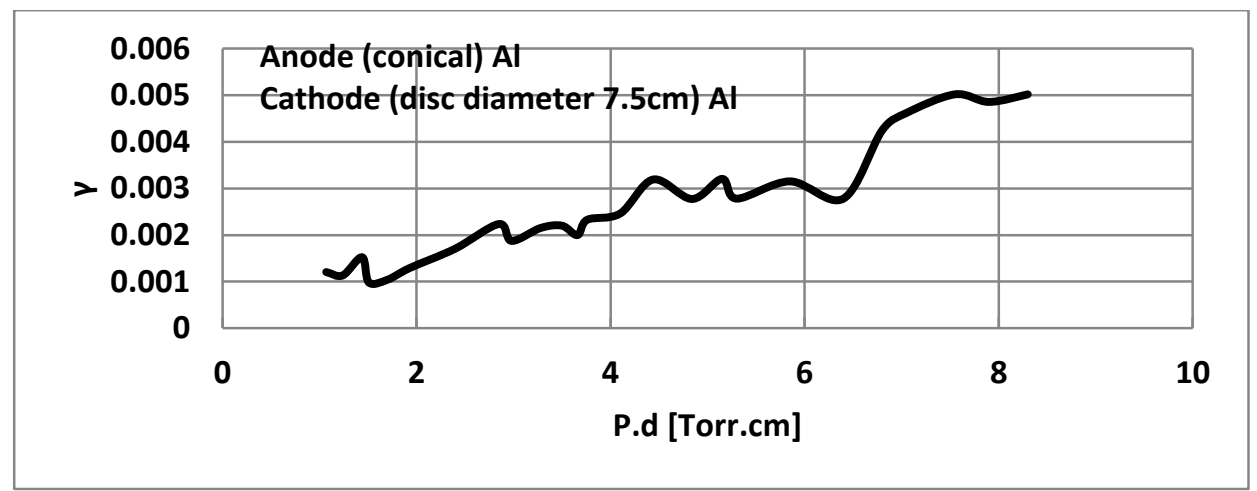

Fig. 13. Secondary Electron Emission Coefficient versus Pd for Mixed Geometry Disc-Conical Electrodes.

Figure (14) shows comparison of secondary electron emission coefficient, $\gamma$, versus Pd for mixed geometry disc-conical electrodes at different pressures. It is obvious that $\gamma$ increases by increasing the nitrogen gas pressure until reaching 0.4 and 0.005 at pressure equal to 0.83 Torr for conical cathode and disc cathode respectively.

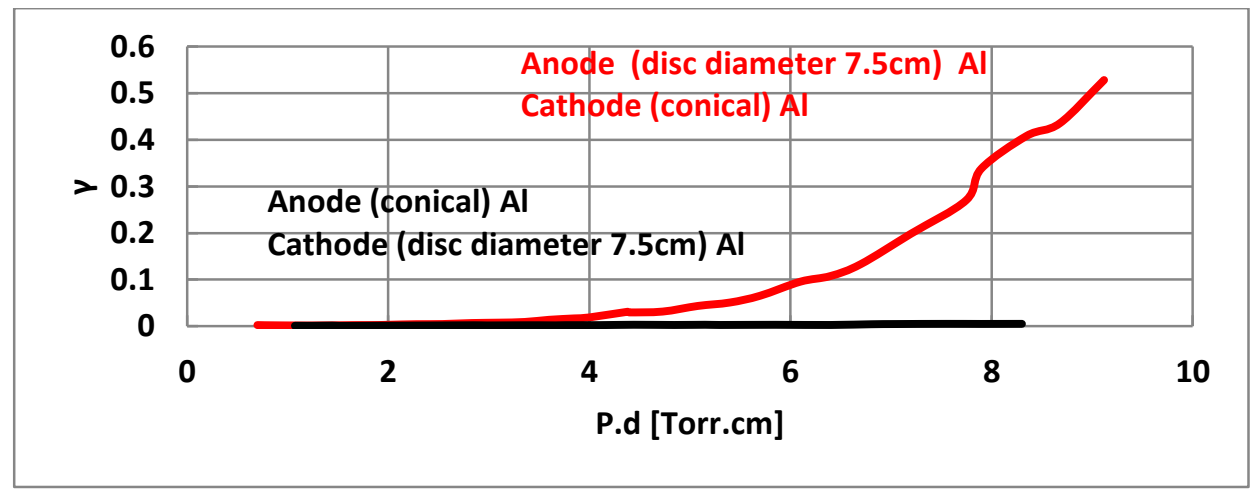

Fig. 14. Secondary Electron Emission Coefficient versus Pd for Mixed Geometry Disc-Conical Electrodes. 


\section{Conclusion}

Practically the entire discharge voltage is concentrated in front of the cathode, in the cathode fall. The electrons are generated from the cathode surface by secondary emission due to energetic ions and fast neutrals bombardment. These electrons are accelerated in the potential drop in front of the cathode and form an electron beam. The net ionization probability per electron is so small that the electrons extracted from the cathode move through the cathode fall region almost without collision. Most of the ionizing processes take place very close to the cathode surface, where the electron energies are rather low and the ionization cross-section has a significant value.

The breakdown voltage has been measured for nitrogen gas discharge using mixed geometry disc-conical electrodes. The minimum breakdown voltage for the two different cathodes shapes occurs at the value of $(\mathrm{Pd})_{\min }$ equal to 1.2 Torr.cm for disc cathode and at $(\mathrm{Pd})_{\min }$ equal to 2 Torr.cm for conical cathode. The minimum breakdown voltage through the plasma means highest ionization cross section. From the Paschen curves for nitrogen gas, it is clear that the minimum breakdown voltage using conical cathode is lower than that disc cathode. This can be attributed to the higher efficiency of secondary ionization processes of disc cathode than of conical cathode.

The secondary electron emission coefficient using the measured values of the breakdown voltages and the first ionization coefficient for discs and conical cathodes has been estimated. It can be concluded that $\gamma$ increases by increasing the nitrogen gas pressure until reaching 0.55 and 0.005 for mixed geometry disc-conical electrodes at pressure equal to 0.9 Torr and 0.8 Torr respectively. Also, it can be concluded that the values of secondary electron emission coefficient of conical cathode are higher than of disc cathode due to higher ions density on conical cathode than that disc one.

\section{References}

[1] Bogaerts, A., Neyts, E., Gijbels, R. and Mullen, J., "Gas Discharge Plasmas and their Applications," ", Spectrochimica Acta Part B, " 57, 2002, pp. 609-658.

[2] Lisovskiy, V.A., Yakovin, S.D. and Yegorenkov, V.D., "Low-Pressure Gas Breakdown in Uniform dc Electric Field," ", J. Phys. D: Appl. Phys.," 33, 21, 2000, pp. 2722-2730.

[3] Berzak, L.F., Dorfman, S.E. and Smith, S.P., "Paschen's Law in Air and Noble Gases", 2006, accessed February 27, 2017, http: //wwweng.lbl.gov/ shuman/XENON/REFERENCES\&OTHER_MISC/paschen_report.pdf.

[4] Saudy, M.A., Eatah, A.I. and Masoud, M.M., "Characteristics of Dc Pseudo-Electric (Virtual Cathode) Discharge in Helium Gas," ", Fizika A14,"” 3, 2005, pp. 225-232.

[5] Bogaerts, A. and Gijbels, R., "The Ion-and Atom-Induced Secondary Electron Emission Yield: Numerical Study for the Effect of Clean and Dirty Cathode Surfaces,", ", Plasma Source Sci. Technol. , " 11, 2002, pp. 27-36.

[6] Townsend, J.S. (ed.), The Theory of Ionization of Gases by Collision, $1^{\text {st }}$. ed., Constable, London, 1910.

[7] Maric, D., Savic, M., Sivos, J., Skoro, N., Radmilovic-Radjenovic, M., Malovic, G., and Petrovic, Z. Lj., "Gas Breakdown and Secondary Electron Yields," ", Eur. Phys. J. D, 68, 2014, 155-161.

[8] Fridman, A. and Kennedy, L.A. (ed.), Plasma Physics and Engineering, ${ }^{\text {st }}$. ed., Taylor \& Francis, New York, London, 2004. 
[9] Radmilovic-Radjenovic, M., Radjenovic, B., Klas, M., Bojarov, A. and Matejcik, S., "The Breakdown Mechanisms in Electrical Discharges: the Role of the Field Emission Effect in Direct Current Discharges in Microgaps," ", Acta Physics, " 63, 3, 2013, pp. 105-205.

[10] Auday, G., Guillot, Ph., Faly, J. and Brunet, H., "Experimental Study of the Effective Secondary Emission Coefficient for Rare Gases and Copper Electrodes," ", J. Appl. Phys., 83, 1998, pp. 5917-21.

[11] Phelps, A.V. and Petrovic, Z.Lj., "Cold-cathode discharges and breakdown in argon: surface and gas phase production of secondary electrons," ", Plasma Sources Sci. Technol., 8, 1999, pp. R21-R44.

[12] Garamoon, A.A., Samir, A., Alakshar, F.F., Kotop, E.F., "Electrical Characteristics of a DC Glow Discharge,",, Plasma Sources Sci. Technol., 12, 2003, pp. 417-420.

[13] Pace, J.D. and Parker, A.B., "The Breakdown of Argon at Low Pressure," ", J. Phys. D: Appl. Phys., 6, 1973, pp. 1525-36.

[14] Phelps, A.., Petrovic, Z.Lj. and Jelenkovic, B.M., "Oscillations of Low-Current Electrical Discharges between Parallel-Plane Electrodes III. Models," ", Phys. Rev. E, 47, 1993, pp. 2825-2838. 